\title{
CHINA IN NUMBERS
}

By 2013 weighted fractional count, China is the second leading country for high-quality science output. Where that research takes place, and who China collaborates with, are shown below.

\section{COUNTRY COLLABORATIONS}

The diagram shows the leading countries by WFC, along with the distribution of their subject strengths and the proportion of their WFC derived from collaborations with mainland China. In the centre, the size and proportion of China's WFC and subject strengths are also shown. Note, this diagram shows all instances of bilateral connections, therefore papers that involve collaborators from more than one country will be double-counted for China.

CHINA'S WFC

TOTAL: 5,206

CHINA-ONLY PAPERS: 4.051

INTERNATIONAL COLLABORATIONS: 1,155

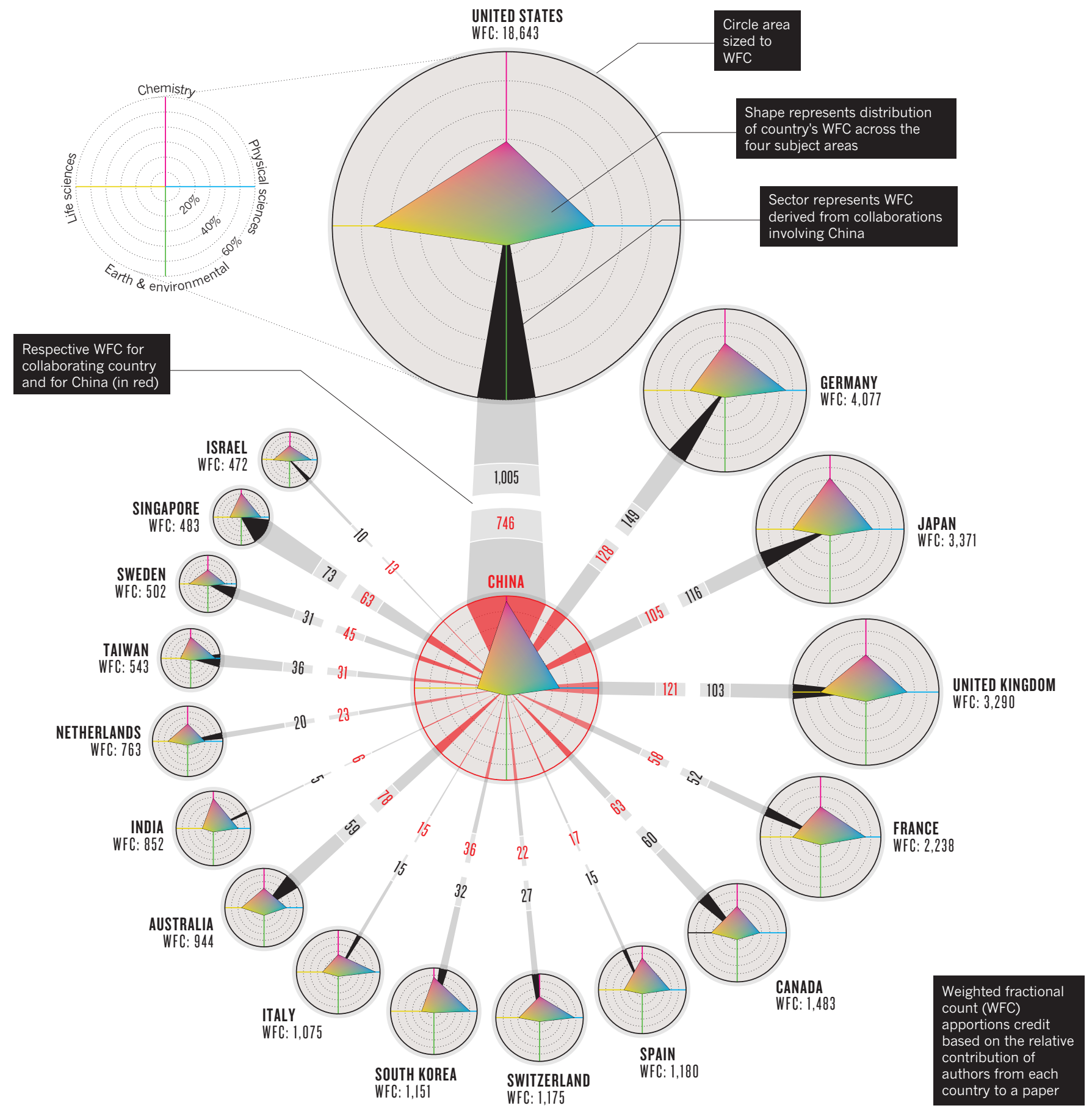




\section{CITY STORY}

The ten leading cities by WFC are shown for mainland China. The solid bubbles are scaled to the combined WFC for all the city's research institutions, including the institutes of the Chinese Academy of Sciences (CAS). Shown for comparison are the WFCs without the CAS institutes (circle outlines). For an analysis of CAS, see page S56.
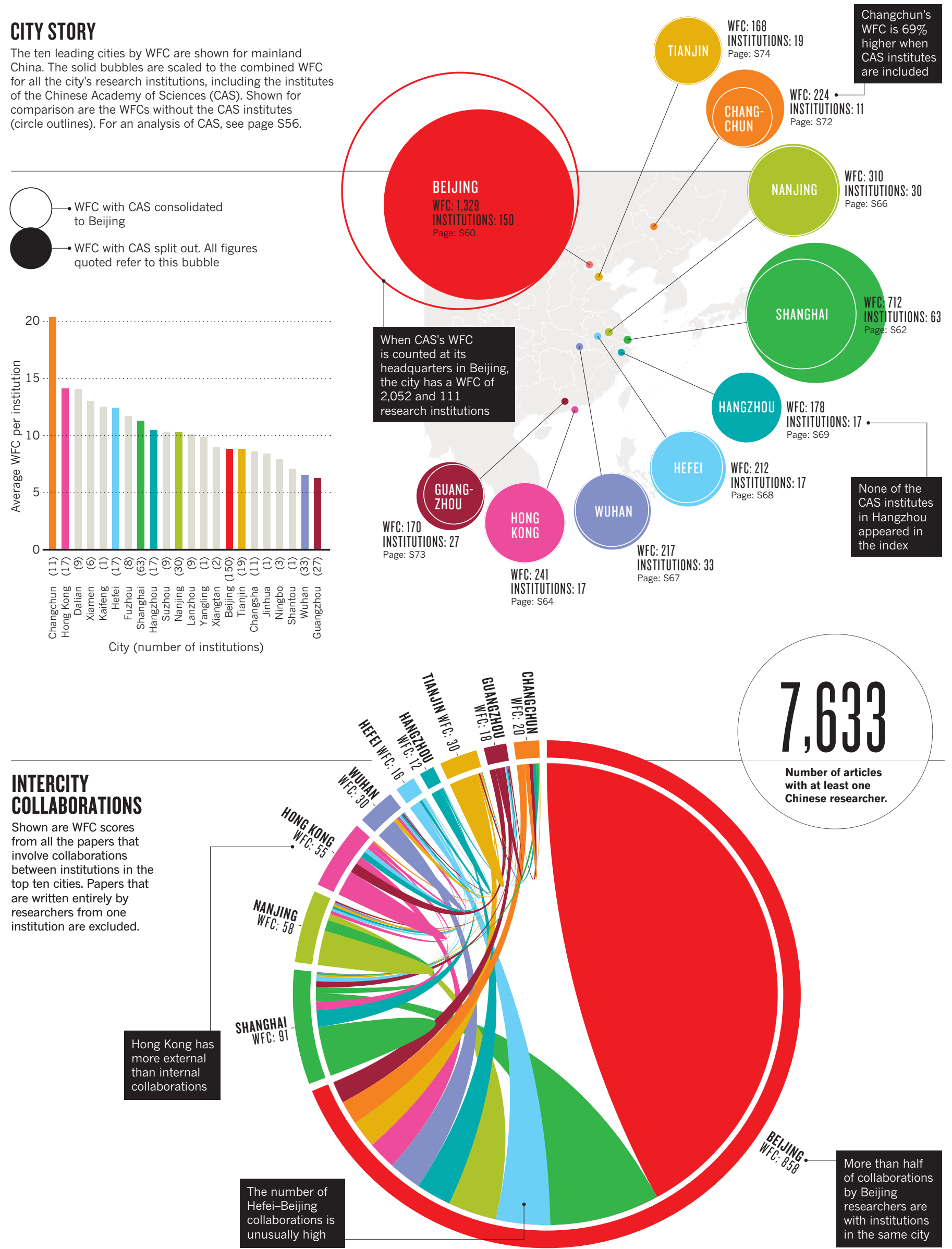